\title{
RADIOTERAPIA COMO TRATAMENTO EXCLUSIVO NO CÂNCER AVANÇADO DA LARINGE
}

\author{
RADIOTHERAPY ALONE FOR ADVANCED LARYNGEAL CANCER
}

\author{
Mauro Marques Barbosa, TCBC-RJ ${ }^{1}$ \\ Fernando Luiz Dias, TCBC-RJ' ${ }^{2}$ \\ Jacob Kligerman, TCBC-RJ ${ }^{3}$ \\ Terence Pires de Faria ${ }^{4}$ \\ Maurílio J. Chagas ${ }^{4}$ \\ Marlos da Paz Leôncio ${ }^{4}$
}

\begin{abstract}
RESUMO: Caracteristicamente os estágios avançados (III e IV) do câncer da laringe são tratados inicialmente por cirurgia, seguida de radioterapia complementar. Esta tem sido a combinação terapêutica que tem obtido os melhores resultados, com relação à maior sobrevida e ao maior tempo livre de doença, nestes pacientes. Porém, como freqüentemente a cirurgia a ser adotada nestes casos representa uma mutilação, perda da voz laríngea, nem sempre esta opção de tratamento é aceita pelos pacientes. Por outro lado, pacientes portadores de doença em estágio avançado podem encontrar-se com suas condições clínicas bastante comprometidas, o que impossibilita a realização de uma cirurgia de grande porte. A associação de quimioterapia e radioterapia tem tido pouca utilidade neste tipo de pacientes, pois geralmente apresentam estado clínico geral comprometido e não suportam a alta toxicidade dos esquemas terapêuticos empregados. Nestes casos a indicação de radioterapia exclusiva, como forma de tentar controlar a doença, tem sido a única arma terapêutica que nos resta, e cujos resultados sempre foram uma incógnita no nosso meio. Foram estudados 62 pacientes portadores de carcinoma epidermóide avançado (estágios III e IV) da laringe, tratados por radioterapia exclusiva, no Hospital do Câncer (INCa), durante os anos de 1992 e 1993. Foi realizado tratamento radioterápico com intenção curativa, sendo empregada a dose clássica de 50cGy em cinco semanas. Estes pacientes foram retrospectivamente analisados quanto à sobrevida, ao estágio da doença, à presença de linfonodos cervicais metastáticos, à idade e à necessidade de traqueostomia prévia. Além da constatação da total ineficácia da radioterapia no controle da doença laríngea estágio IV, detectou-se que a sobrevida de três anos igual a 54\% para os pacientes do estágio III e a preservação da função da laringe em $2 / 3$ dos pacientes, poderão representar, dependendo de estudos mais abrangentes, uma alternativa terapêutica para pacientes selecionados.
\end{abstract}

Unitermos: Câncer da Laringe; Radioterapia.

\section{INTRODUÇÃO}

O tratamento do câncer avançado da laringe representa um grande desafio para o cirurgião de cabeça e pescoço, pois este é defrontado com a necessidade de controlar uma doença muito agressiva, enquanto que, por outro lado, sua opção terapêutica mais eficaz é uma intervenção cirúrgica mutilante com a perda da voz.

Outras vezes nos deparamos com pacientes que não aceitam o tratamento cirúrgico proposto, ou mesmo não têm as condições clínicas necessárias para se submeterem a ele.

1. Titular da Seção de Cirurgia de Cabeça e Pescoço do Hospital do Câncer (INCa). Fellow of the American College of Surgeons (FACS)

2. Chefe da Seção de Cirurgia de Cabeça e Pescoço do Hospital do Câncer (INCa), Fellow of the American College of Surgeons (FACS)

3. Diretor Geral do Instituto Nacional de Câncer (INCa), Fellow of the American College of Surgeons (FACS)

4. Médico Residente da Seção de Cirurgia de Cabeça e Pescoço do Hospital do Câncer (INCa)

Recebido em 8/12/99

Aceito para publicação em 29/5/2000

Trabalho realizado na Seção de Cirurgia de Cabeça e Pescoço do Hospital do Câncer (INCa) - Rio de Janeiro-RJ 
Também a alternativa de utilização da combinação de radioterapia com quimioterapia, preconizada em alguns protocolos de preservação de órgão, não tem sido, em nosso grupo de pacientes, muito bem-sucedida, pois estes encontram-se freqüentemente sem as condições clínicas necessárias para esta abordagem terapêutica.

Este trabalho foi realizado com o objetivo de avaliarmos os resultados terapêuticos obtidos com os pacientes portadores de carcinoma laríngeo avançado, e que, por qualquer motivo, não foram submetidos ao tratamento cirúrgico padronizado, mas sim à radioterapia exclusiva, buscando inclusive detectar os fatores patológicos que influenciaram na sobrevida destes pacientes, e o índice de preservação da função laríngea com a radioterapia curativa usada isoladamente nos tumores T3 e T4 da laringe.

\section{PACIENTES E MÉTODOS}

Foram analisados, retrospectiva e aleatoriamente, 62 pacientes portadores de carcinoma epidermóide da laringe, estágios III e IV, atendidos na Seção de Cirurgia de Cabeça e Pescoço do Hospital do Câncer (INCa), nos anos de 1992 e 1993, e que foram submetidos à radioterapia exclusiva.

As indicações da radioterapia exclusiva foram devidas à recusa do paciente a submeter-se ao tratamento cirúrgico, falta de condições clínicas para a cirurgia, ou mesmo à irressecabilidade da lesão tumoral.

O tratamento radioterápico foi feito com intenção curativa em todos os pacientes, sendo empregada a dose clássica de 50cGy em cinco semanas.
Como fatores que poderiam influenciar na sobrevida dos pacientes foram analisados: o estágio da doença, a presença de linfonodos cervicais positivos, a idade do paciente, e a necessidade de realização de traqueotomia prévia.

O tempo médio de seguimento destes pacientes foi de 22 meses.

Como indicativo de função laríngea eficiente foi usada a taxa de decanulização dos pacientes previamente traqueotomizados, associada ao número de pacientes que não necessitaram de traqueotomia durante o curso do tratamento.

Os testes estatísticos empregados neste trabalho foram: Kaplan-Meier para a análise da sobrevida, Wilcoxon-Gehan para avaliação univariada dos fatores estudados e o Cox Regression para análise multivariada dos fatores incidentes.

\section{RESULTADOS}

Quanto ao sexo, tivemos que $84 \%$ dos pacientes eram do sexo masculino e $16 \%$ do feminino, o que representou uma incidência de 5,25/1. A idade variou entre 34 e 81 anos, com a média de 59 anos (Figura 1).

Com respeito aos hábitos sociais, encontramos $84 \%$ de etilistas e $94 \%$ de tabagistas (Figura 2).

Neste levantamento tivemos 20 pacientes classificados como estágio III $(32,3 \%)$ e 42 pacientes no estágio IV $(67,7 \%)$, sendo que $60 \%$ de todos os pacientes tiveram necessidade de serem submetidos à traqueotomia pré-tratamento (Figura 3).

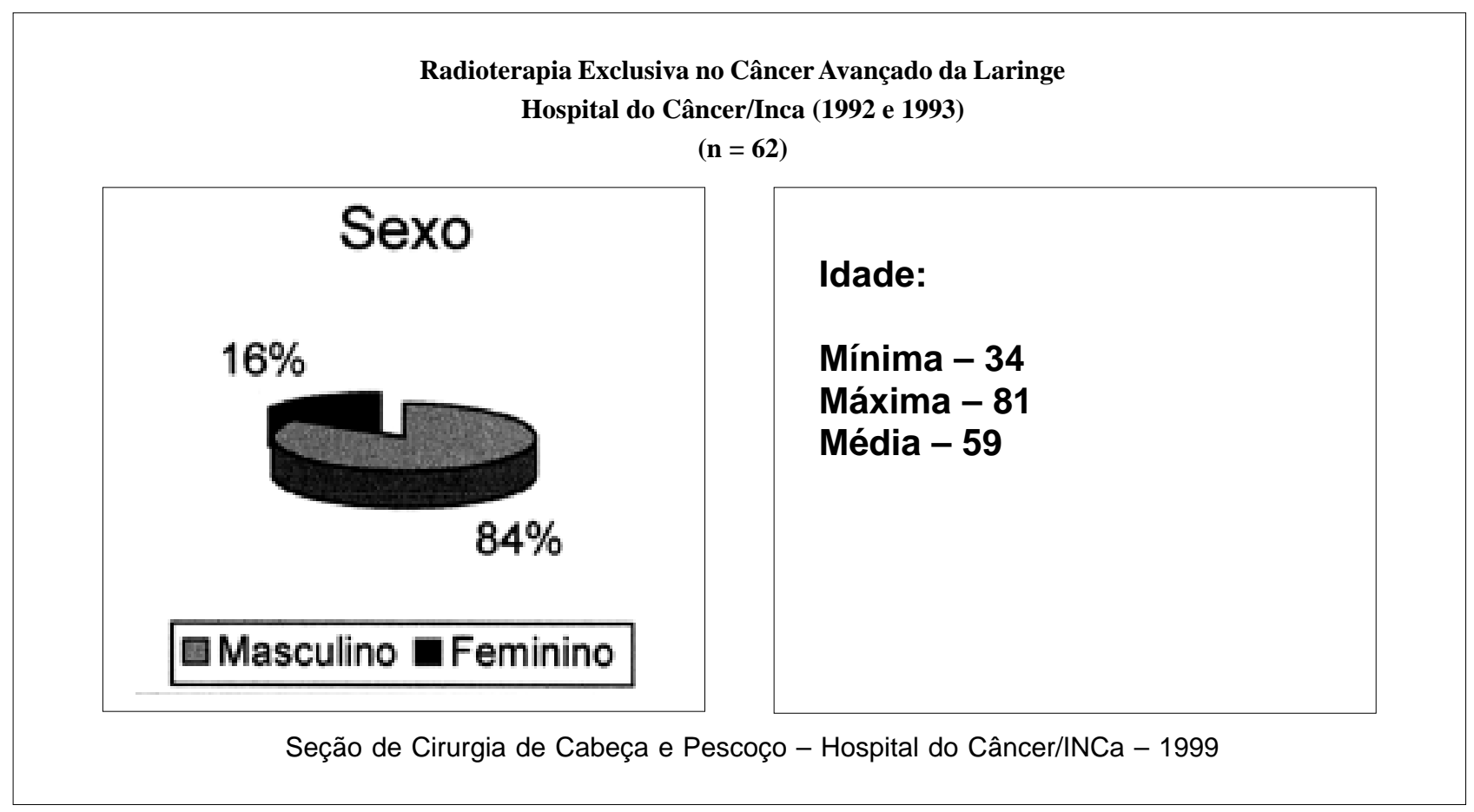

Figura 1 - Distribuição do sexo e idade dos pacientes estudados. 


\section{Radioterapia Exclusiva no Câncer Avançado da Laringe}

Hospital do Câncer/Inca (1992 e 1993)

$(n=62)$
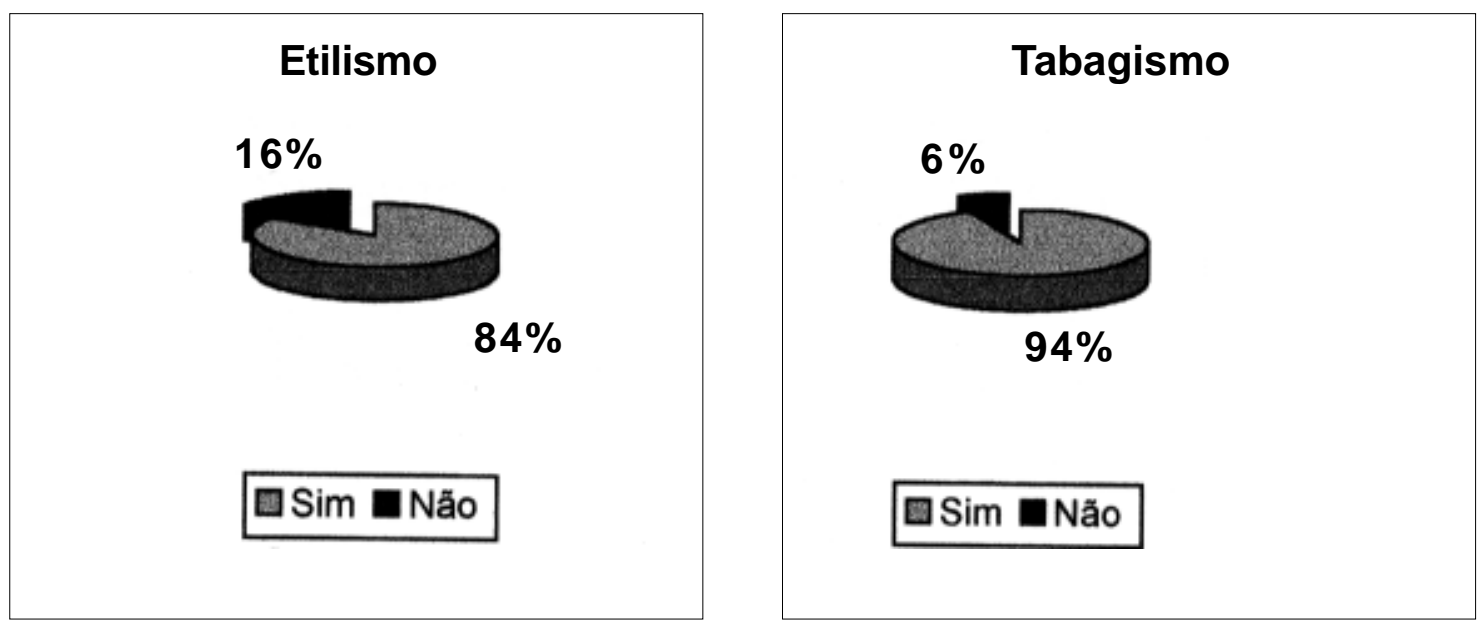

Seção de Cirurgia de Cabeça e Pescoço - Hospital do Câncer/INCa - 1999

Figura 2 - Hábitos sociais dos pacientes avaliados.

Durante o seguimento foram detectadas metástases a distância em $8 \%$ dos pacientes, sendo que os órgãos mais freqüentemente atingidos foram o pulmão e os ossos. Além disso, 4,8\% dos casos evoluíram com a presença de segundo tumor primário, geralmente no pulmão (Figura 4).

Como melhor resultado obtido tivemos que os pacientes estágio III apresentaram sobrevida de 36 meses igual a $54 \%$, sendo que $66 \%$ destes pacientes puderam ser decanulizados (ou seja não mais necessitaram da traqueotomia), o que significou o controle da doença e a preservação do órgão (laringe funcionante) em $2 / 3$ dos pacientes estágio III (Figura 5).

As análises estatísticas realizadas demonstraram que a diferença na sobrevida de três anos dos pacientes estágio

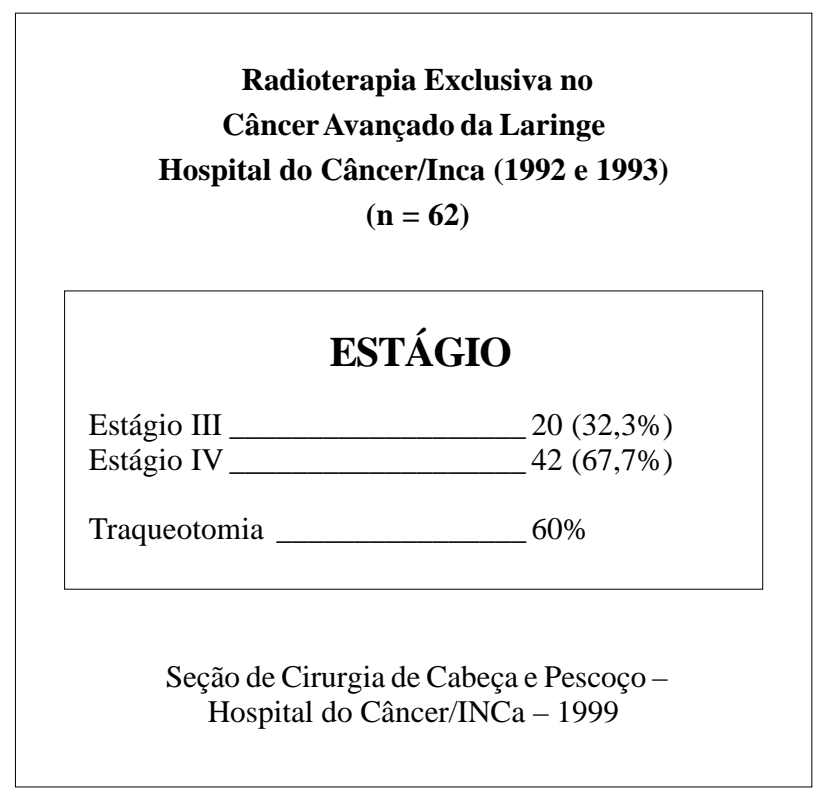

Figura 3 - Distribuição dos estágios estudados e taxa de traqueotomia prévia.

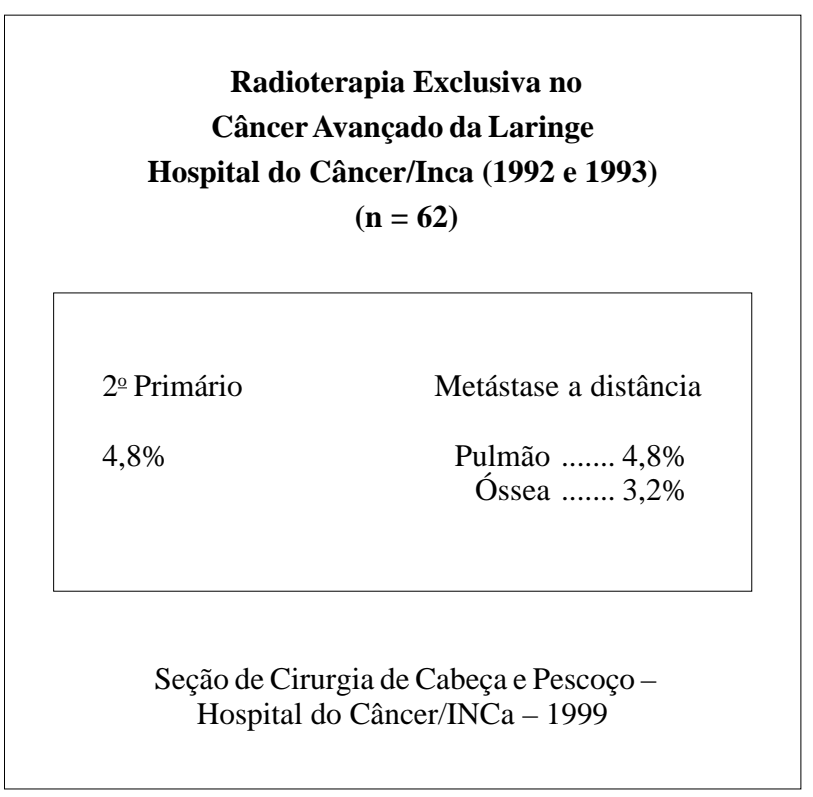

Figura 4-Incidência de segundo tumor primário e de metástases a distância. 


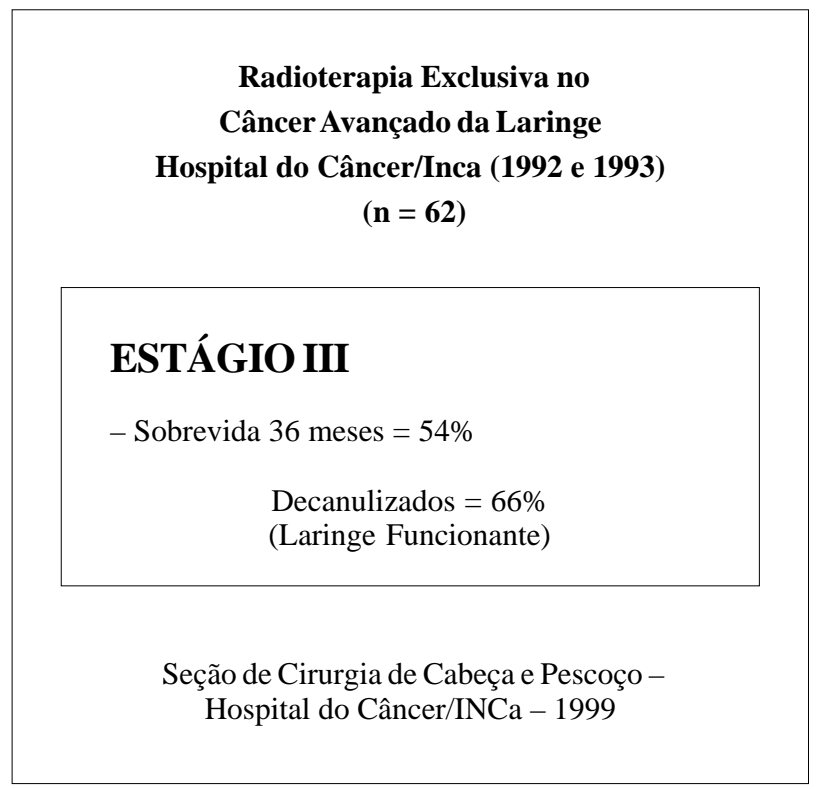

Figura 5 - Índice de sobrevida dos pacientes estágio III e taxa de preservação do órgão nestes pacientes.

III (54\%) quando comparada com aquela dos pacientes estágio IV $(9,5 \%)$ foi significativa na análise univariada $(p=0,01)$, porém não teve significância na multivariada.

A necessidade da realização da traqueotomia prévia piorou a sobrevida dos pacientes (13\% contra $47 \%)$, o que foi significativo na análise univariada $(\mathrm{p}=0,01)$, mas sem significado na multivariada (Figura 6).

A presença de linfonodos cervicais positivos fez com que nenhum dos pacientes sobrevivesse três anos, enquanto que os que não tinham linfonodos metastáticos tiveram uma sobrevida de $39 \%$. Esta diferença só foi significante no estudo univariado $(\mathrm{p}=0,03)$.

Embora nenhum dos nossos pacientes com idade menor do que 45 anos tenha atingido os 36 meses de sobrevida, enquanto nos mais velhos esta sobrevida foi de $20 \%$, esta diferença nada representou em termos estatísti$\cos$ (Figura 7).

\section{DISCUSSÃO}

A busca de soluções alternativas para o tratamento do câncer avançado da laringe tem sido uma constante nos Serviços de Cirurgia de Cabeça e Pescoço, seja na tentativa de oferecer-se opções aos pacientes que se recusam à terapia cirúrgica, seja para aqueles que não apresentam condições clínicas gerais de se submeterem a tal procedimento $^{1-13}$.

Nos últimos anos temos observado uma tentativa de ampliação das indicações das cirurgias parciais, originariamente descritas para lesões em estágios mais iniciais da doença. Assim, tivemos a grande difusão das laringectomias parciais, das supracricóides e mesmo da near-total, todas objetivando controlar a neoplasia laríngea, mas conservando algum tipo de comunicação vocal ${ }^{14-22}$.

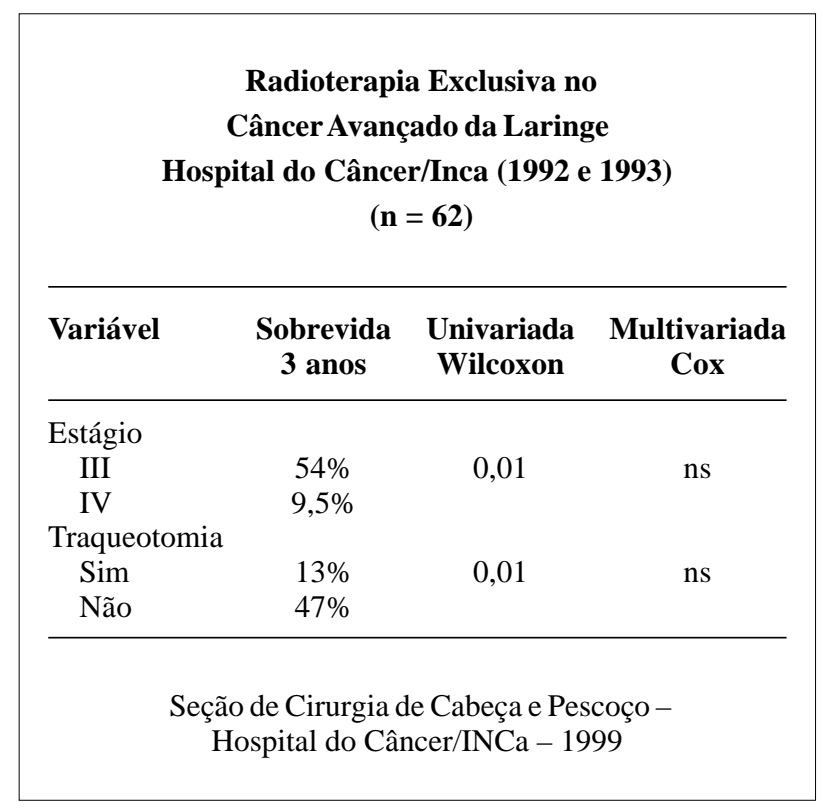

Figura 6-Cálculos estatísticos do estágio e traqueotomia prévia.

\begin{tabular}{|c|c|c|c|}
\hline \multicolumn{4}{|c|}{$\begin{array}{c}\text { Radioterapia Exclusiva no } \\
\text { Câncer Avançado da Laringe } \\
\text { Hospital do Câncer/Inca (1992 e 1993) } \\
\text { (n = 62) }\end{array}$} \\
\hline Variável & $\begin{array}{l}\text { Sobrevida } \\
3 \text { anos }\end{array}$ & $\begin{array}{l}\text { Univariada } \\
\text { Wilcoxon }\end{array}$ & $\begin{array}{l}\text { Multivariada } \\
\text { Cox }\end{array}$ \\
\hline $\begin{array}{l}\text { Linfonodo } \\
\text { Sim } \\
\text { Não }\end{array}$ & $\begin{array}{c}0 \\
39 \%\end{array}$ & 0,03 & ns \\
\hline $\begin{array}{l}\text { Idade } \\
\quad<45 \\
\quad>45\end{array}$ & $\begin{array}{c}0 \\
20 \%\end{array}$ & ns & ns \\
\hline \multicolumn{4}{|c|}{$\begin{array}{l}\text { Seção de Cirurgia de Cabeça e Pescoço - } \\
\text { Hospital do Câncer/INCa - } 1999\end{array}$} \\
\hline
\end{tabular}

Figura 7-Análise estatística dos fatores linfonodos positivos e idade.

Por outro lado, a associação de quimioterapia e radioterapia na tentativa de preservação da laringe, que apresentou em alguns centros resultados bastante promissores $^{23,24,25}$, tem se mostrado, em nossos pacientes, pouco encorajadora, devido à grande toxicidade dos esquemas utilizados e às más condições clínicas destes pacientes.

O resultado de 54\% de sobrevida em três anos para pacientes com câncer da laringe estágio III submetidos à radioterapia exclusiva, embora um pouco pior do que aquele que nós mesmos temos conseguido com os pacientes deste 
estágio tratados por laringectomia total (80\%), faz com que possamos pensar na radioterapia como uma alternativa válida para alguns pacientes.

Tão importante quanto este resultado de sobrevida foi o obtido na preservação da laringe como um órgão funcionante, aspecto que vem sendo ressaltado na literatura mundial $^{26-31}$, e que no presente estudo representou a significativa cifra de $2 / 3$ dos pacientes que puderam ser decanulizados de suas traqueotomias ou dela não precisaram em nenhuma fase do tratamento.

A chave para obtenção de bons resultados está na criteriosa seleção dos pacientes, considerando-se não apenas a extensão local do tumor, mas também sua profundidade, as regiões da laringe acometidas, a presença ou não de metástases linfáticas cervicais, a idade do paciente e suas condições clínicas gerais.

Acreditamos que a opção por radioterapia isolada no tratamento dos tumores avançados da laringe, pode representar uma alternativa válida, muito embora estudos ran- domizados e prospectivos sejam necessários, não só para a confirmação destes resultados, como também para fornecer informações mais detalhadas a respeito das características dos pacientes que mais se beneficiarão desta opção, além de poder ser uma forma de tratamento com potencial de preservação do funcionamento da laringe em pacientes criteriosamente escolhidos.

Deste estudo pudemos então concluir que:

A radioterapia exclusiva foi totalmente ineficaz no controle da doença laríngea no estágio IV.

$\mathrm{O}$ estagiamento da doença, a necessidade de traqueotomia prévia e a presença de linfonodos cervicais metastáticos, foram fatores que isoladamente influenciaram o prognóstico dos nossos pacientes.

Para os pacientes do estágio III é necessário um estudo mais amplo, de preferência prospectivo e randomizado, para podermos avaliar o real benefício desta opção terapêutica, inclusive como conduta capaz de preservar o funcionamento da laringe em pacientes especialmente selecionados.

\begin{abstract}
Advanced-stage (III and IV) laryngeal cancers have been classical treated by a combination of surgery and complementary radiotherapy. As surgery normally represents a mutilation in these cases (loses of laryngeal voice), some patients refuse it. Another group of patients presents such a deteriorated clinical condition that they shall not be submitted to a high-risk surgery. Chemotherapy and radiotherapy association has been less useful in our patients, which are frequently in bad clinical conditions and do not tolerate the therapeutical schemes high toxicity. In these cases, radiotherapy alone is the sole option we have to offer trying to control the disease. The authors retrospectively reviewed 62 advanced-stage (III an IV) laryngeal cancer patients treated by radiotherapy alone at Hospital do Câncer (Rio de Janeiro) during 1992 and 1993. The treatment had a curative intention in all cases with a classical dose of 50cGy in 5 weeks. We analyzed survival, disease stage, cervical metastasis, age, and the need for previous tracheotomy. Statistical analysis showed a 36 months stage III survival of 54\% and 2/3 of them were kept without a tracheotomy tube, suggesting preservation of a functional larynx. This result had encouraged the authors to randomize studies for a better selection of patients who can mostly benefit from this therapeutic option.
\end{abstract}

Key Words: Laringeal Cancer; Radiotherapy.

\section{REFERÊNCIAS}

1. DeSanto LW. T3 Glottic Cancer: Options and Consequences of the Options. Laryngoscope 1984; 94:1311-5.

2. Meredith APD, Randall CJ, Shaw HJ. Advanced Laryngeal Cancer: A Management Perspective. The Journal of Laryngology and Otology 1987; 101:1046-54.

3. Sandberg N, Mercke C, Turesson I. Glottic Laryngeal Carcinoma With Fixed Vocal Cord Treated With Full-Dose Radiation, Total Laryngectomy or Combined Treatment. Acta Oncologica 1990; 29: 509-11.

4. Terhaard CHJ, Boer MF, Croll GA, et al. Prognostic Factors for Survival in Patients With T3 Laryngeal Carcinoma. Am J Surg 1992; 164: 682-7.

5. Foote RL, Olsen KD, Buskirk SJ et al. Laryngectomy Alone for T3 Glottic Cancer. Head \& Neck Surgery 1994; 16: 406-12.

6. Levine PA, Brasnu DF, Ruparelia A et al. Management of advanced-stage laryngeal cancer. Otolaryngol Clin. North Am. 1997; 30: 101-12.
7. Porter MJ, McIvor NP, Morton RP et al. Audit in the management of T3 fixed-cord laryngeal cancer. Am J Otolaryngol 1998; 19:360-4.

8. Lo SM, Venkatesan V, Matthews TW et al. Tumor volume: implications in T2/T3 glottic/supraglottic squamous cell carcinoma. J Otolaryngol 1998; 27:247-51.

9. Kaanders JH, Pop LA, Marres HA, et al. Accelerated radiotherapy with carbogen and nicotinamide (ARCON) for laryngeal cancer. Radiother Oncol 1998; 48:115-22.

10. Vallicioni JM, Giovanni A, Triglia JM et al. Laryngeal cancer in a young adult. Presse Med 1999; 1-8;28: 908-10

11. Tkachev SI, Alieva SB, Alferov VS. Standard and hyperfractionated irradiation for locally advanced tumors (T3N0M0) of the larynx. Vopr Onkol 1999; 45: 305-8.

12. Golen M, Skladowski K, Maciejewski B. Comparative estimation of cure rates for supraglottic and glottic cancer in radiotherapy. Neoplasma 1999; 46: 66-71.

13. MacKenzie RG; Franssen E; Balogh JM et al. Comparing treatment outcomes of radiotherapy and surgery in 
locally advanced carcinoma of the larynx: a comparison limited to patients elegible for surgery. Int. J. Radiat. Oncol. Biol. Phys. 2000; 47: 65-71.

14. Bocca E, Pignataro O, Oldini C et al. Extended Supraglottic Laryngectomy-Review of 84 Cases. Ann Otol Rhinol Laryngol 1987; 96: 384-6.

15. Laccourreye H, Guily JLS, Brasnu D et al. Supracricoid Hemilaryngopharyngectomy - Analysis of $240 \mathrm{Ca}-$ ses. Ann Otol Rhinol Laryngol 1987; 96: 217-21.

16. Laccourreye H, Laccourreye O, Weinstein G, et al. Supracricoid Laryngectomy With Cricohyoidopexy: A Partial Laryngeal Procedure for Selected Supraglottic and Transglottic Carcinomas. Laryngoscope 1990; 100: 735-41.

17. Laccourreye H, Laccourreye O, Weinstein G et al. Supracricoid Laryngectomy With Cricohyoidoepiglottopexy: A Partial Laryngeal Procedure For Glottic Carcinoma. Ann Otol Rhinol Laryngol 1990; 99: 421-6.

18. Piquet J, Chevalier D. Subtotal Laryngectomy With CricoHioido-Epiglotto Pexy for the Treatment of Extended Glottic Carcinomas. Am J Surg 1991; 162: 357-61.

19. Levine PA, Debo RF, Reibel JF. Pearson Near-Total Laryngectomy: A Reproducible Speaking Shunt. Head \& Neck Surgery 1994; 16: 323-5.

20. Vincentiis M, Minni A, Gallo A. Supracricoid Laryngectomy With Cricohyoidopexy (CHP) in the Treatment of Laryngeal Cancer: A Functional and Oncologic Experience. Laryngoscope 1996; 106: 1108-14.

21. Lima RA, Freitas EQ, Kligerman J, et al. Near-Total Laryngectomy for Treatment of Advanced Laryngeal Cancer. Am J Surg 1997; 174: 490-1.

22. Song F; Zhang Q; Wang J. Partial laryngectomy for treatment of the laryngeal cancer of T3 and T4 categories. Chung Hua Erh Pi Yen Hou Ko Tsa Chih 1997; 32 : 342-344.

23. Fu KK. Radiation Therapy With 5-fluorouracil in Head and Neck Cancer. Semin Radiat Oncol 1997; 7: 274-282.

24. Carew JF, Shah JP. Advances in multimodality therapy for laryngeal cancer. CA Cancer J Clin 1998; 48: 211-28.

25. Chougule PB, Akhtar MS, Akerley W et al. Chemoradiotherapy for advanced inoperable head and neck cancer: A phase II study. Semin Radiat Oncol 1999; 9(Suppl 1): 58-63.
26. DeSanto LW, Olsen KD, Perry WC et al. Quality of Life After Surgical Treatment of Cancer of the Larynx. Ann Otol Rhinol Laryngol 1995; 104: 763-9.

27. de Graeff A, de Leeuw RJ, Ros WJ et al. A prospective study on quality of life of laryngeal cancer patients treated with radiotherapy. Head Neck 1999; 21: 291-6.

28. Finizia C, Hammerlid E, Westin T et al. Quality of life and voice in patients with laryngeal carcinoma: a posttreatment comparison of laryngectomy (salvage surgery) versus radiotherapy. Laryngoscope 1998; 108: 1566-73.

29. Finizia C, Dotevall H, Lundstrom E et al. Acoustic and perceptual evaluation of voice and speech quality: a study of patients with laryngeal cancer treated with laryngectomy vs irradiation. Arch Otolaryngol Head Neck Surg 1999; 125: 157-63.

30. Allal AS, Dulguerov P, Bieri S et al. Assessment of quality of life in patients treated with accelerated radiotherapy for laryngeal and hypopharyngeal carcinomas. Head Neck 2000; 22: 288-293.

31. Leon X, Quer M, Orus C et al. How much does it cost to preserve a larynx? An economic study. Eur Arch Otorhinolaryngol 2000; 257(2): 72-76.

\section{ENDEREÇO PARA CORRESPONDÊNCIA:}

Dr. Mauro Marques Barbosa

Av. N. S. Copacabana, 897/1102

22060-000 — Rio de Janeiro-RJ

E-mail: maurobarbosa@windnet.psi.br 